DOI: $\underline{\text { https://doi.org/10.24867/07OI01Dimic }}$

\title{
SISTEM ZA BEZBEDNU KOMUNIKACIJU DVA KORISNIKA PUTEM RAZMENE PORUKA
}

\section{SECURE MESSAGING COMMUNICATION BETWEEN TWO USERS}

\author{
Slađana Dimić, Fakultet tehničkih nauka, Novi Sad
}

\section{Oblast - INŽENJERSTVO INFORMACIONIH SISTEMA}

\begin{abstract}
Kratak sadržaj - Cilj ovog rada jeste da se obezbedi sigurna komunikacija u vidu razmene mail-a dva korisnika preko Web aplikacije i Microsoft Outlook-a, klijenta Microsoft Exchange-a. Za ovu komunikaciju potrebno je registrovati novu aplikaciju na Microsoft Azure portalu koja ima identifikatore neophodne za dobavljanje Bearer tokena. Bearer token sluzi da se klijent, u ovom slučaju razvijena Web aplikacija, autentifikuje servisu. Klijent je tada u mogućnosti da, preko Microsoft Graph RESTful Web API-a, potraži sve korisnike određene grupe registrovane na Azure Active Directory-u i pošalje mail odabranom korisniku.
\end{abstract}

Ključne reči: Exchange server, Graph API, Bearer token, RESTful servis, sigurna komunikacija, autorizacija Abstract - The aim of this thesis is to provide secure communication in the form of an email exchange between two users via Web application and Microsoft Outlook, a Microsoft Exchange client. This communication requires a new registered application on the Microsoft Azure portal that has the identifiers that are necessary to deliver the Bearer token. The Bearer token serves to authenticate the client to the service, in this case the client is a developed Web application. The client is then able to, through the Microsoft Graph RESTful API, pull all users of a particular group registered with Azure Active Directory and send mail to the selected user.

Keywords - Exchange server, Graph API, Bearer token, RESTful service, secure communication, authorization

\section{UVOD}

Platformski Microsoft servis Azure Active Directory daje mogućnost kompanijama širom sveta da skladište svoje podatke na Cloud, radi povećanja kapaciteta skladištenja podataka i obezbeđenja sigurnosti podataka. Kompanije tako, pored ostalih podataka, skladište podatke o svim korisnicima, odnosno članovima kompanije. Kako je svaka kompanija podeljena u više sektora, kao što su na primer sektor razvoja, sektor prodaje, sektor finansija i slično, tako svaki korisnik pripada određenoj grupi, odnosno sektoru. Neke kompanije današnjice imaju svoj softver kroz koji vode poslovanje. Kako bi zaposleni kompanije i njeni korisnici komunicirali uz pomoć softvera, odnosno razvijene Web aplikacije slanjem

\section{NAPOMENA:}

Ovaj rad proistekao je iz master rada čiji mentor je bio dr Srđan Sladojević, vanr. profesor. mail-a na željeni Microsoft Outlook nalog, tako da komunikacija bude sigurna operacija, potrebno je registrovati novu aplikaciju na Microsoft Azure portalu i dodeliti joj permisije koje će u nastavku teksta biti opisane. Preko takve registrovane aplikacije, kompanijska Web aplikacija bila bi u mogućnosti da potraži Bearer token koji služi za autentifikaciju servisu. Sa dobijenim Bearer token-om kompanijska Web aplikacija preko Microsoft Graph API-a i RESTful servisa predefinisanim upitom može da potraži sve korisnike određene grupe. Nakon što dobije korisnike, takođe preko Microsoft Graph API-a i RESTful servisa uz pomoć Bearer autentifikacije može da pošalje mail odabranim korisnicima $\mathrm{u}$ ime željenog korisnika. Za povlačenje korisnika određene grupe potreban je identifikator grupe, dok je za slanje mail-a u ime određenog korisnika potreban identifikator korisnika iz Active Directory-a. Ovim pristupom se izbegava lokalno čuvanje podataka korisnika koje zauzima memoriju, obezbeđuje sigurnost podataka kojima ne može svako da pristupi, kao i zloupotreba podataka.

\section{PREGLED RELEVANTNE LITERATURE 2.1. Microsoft Exchange Server}

Microsoft Exchange je u osnovi mail server razvijen od strane Microsoft-a koji, između ostalog, podržava i svoj klijent Outlook. Exchange omogućava korisnicima da razmenjuju informacije pomoću Outlooka ili Outlook Web Accessa, dakle omogućava saradnju među korisnicima. Od verzije Exchange 2000, Exchange više nema svoj directory servis, već se kompletno oslanja na Active Directory infrastrukturu. Exchange je teško konfigurisati pa ga manje firme ne implementiraju, ali ta kompleksnost poseduje funkcije koje su važne za povezivanje nekoliko hiljada Exchange korisnika [1].

\subsection{Microsoft Outlook}

Microsoft Outlook je Microsoft aplikacija za email, koja takođe uključuje kalendar, koordinisanje zadacima, menadžer kontakata, beleške, dnevnik i Web pregled. Može se koristiti kao samostalna aplikacija ili može raditi sa Microsoft Exchange serverom za vise korisnika $\mathrm{u}$ organizaciji [2].

\subsection{Microsoft Azure Active Directory}

Azure je Microsoft platforma za Cloud koja omogućava hostovanje aplikacija i čuvanje dokumenata.

Active directory je servis kreiran od strane Microsoft-a, koji čuva informacije o objektima na mreži tako da im autentifikovani korisnici kao i administratori mreže u svakom trenutku mogu lako pristupiti [3]. 


\subsection{Microsoft Cloud}

Cloud podrazumeva skladištenje podataka na internetu, a ne lokalno na hard disk drive-u. Nije reč samo o podacima, skladište se i kompletni sistemi, potreban je samo izlaz na internet.

\subsection{REST arhitektura}

REST (Representational state transfer) arhitekturalni stil je set principa koji definiše kako bi Web standardi poput HTTP (Hypertext Transfer Protocol) i URI (Uniform Resource Identifier) trebali da se koriste. Ipak, na programerima i arhitektama je da ga održe uniformnim. Nije zavistan ni od jednog protokola, ali gotovo svaki RESTful servis koristi HTTP/HTTPS (Hypertext Transfer Protocol Secure) kao osnovni protokol pa tako, REST označava način komunikacije između klijenta i servera korišćenjem mrežnih resursa pomoću HTTP protokola. Američki naučnik Roj Filding (Roy Fielding) je u svojoj doktorskoj disertaciji [4] prvi put pomenuo pojam REST gde je rekao da je motivacija za razvoj $R E S T$-a pravljenje modela arhitekture koji opisuje kako bi Web trebalo da radi, takvog da može da posluži kao orijentir pri definisanju standarda protokola za Web.

\subsection{RESTful servis}

RESTful servisi su zasnovani na REST arhitekturi i dobro su integrisani sa HTTP protokolom. Ne zahtevaju XML (Extensible Markup Language) poruke kao ni WSDL (Web Services Description Language) definiciju. Prilično je jednostavan za korišćenje (human readable) i važi da dominantan mrežni servis današnjice. Podaci se najčešće razmenjuju u JSON, XML i YAML formatima. Može biti izvršen na bilo kom klijentu ili serveru koji ima HTTP/HTTPS podršku. Kod ovog tipa servisa, resursi (npr. statičke strane, fajlovi, podaci iz baze...) imaju sopstveni URL (Uniform Resource Locator) ili URI (Uniform Resource Identifier) koji ih identifikuju. Pristup do resursa je definisan HTTP protokolom, gde svaki poziv čini jednu akciju (kreira, čita, menja ili briše podatke). Isti $U R L$ se koristi za sve operacije ali se menja HTTP metod koji definiše vrstu operacije. REST koristi Create, Read, Update, Delete (CRUD) HTTP metode kao što su: GET, POST, PUT, DELETE [5]. Skoro svi veliki Web servisi kao što su Google, Twitter i Facebook se oslanjaju na REST arhitekturu za njihov API (Application programming interface), zato što HTTP protokol pokreće skoro sve internet konekcije. Klijent i servis ne moraju da budu u uskoj vezi prilikom razmenjivanja poruka, pa tako sve informacije koje se prenose kao resursi staju u jedinstveni identifikator URI koji je kao takav dovoljan za razumevanje zahteva. Ukoliko klijent i servis žele da komuniciraju na siguran način, Header sekcija HTTP zahteva podržava vid autorizacije putem Bearer token-a koji Microsoft obezbeđuje, što predstavlja jedan od načina sigurne komunikacije.

\subsection{Microsoft Graph API}

Microsoft Graph je RESTful Web API koji omogućava pristup resursima Microsoft Cloud usluge. Nakon što se registruje aplikacija i dobije token za autentifikaciju za korisnika ili uslugu, mogu se podnositi zahtevi za Graph API. Graph API-u se pristupa HTTP metodama, najčešće su POST, GET, PUT i DELETE metode koje opisuju CRUD (Create, Read, Update, Delete) operacije [5].

\subsection{Bearer autentifikacija}

Bearer autentifikacija kao provera identiteta nosioca, odnosno provera identiteta token- $a$, je HTTP šema provere autentičnosti Bearer token-a. Bearer autentifikacija se može shvatiti i kao davanje pristupa nosiocu ovog token-a. Bearer token je kriptovani string koji je uglavnom generisan od strane servera kao odgovor na zahtev. Klijent dobijeni token šalje kroz zaglavlje autorizacije prilikom definisanja HTTP zahteva [6].

\section{OPIS FUNKCIONALNOSTI}

U ovom poglavlju će biti predstavljena registracija nove aplikacije na Microsoft Azure portalu, koja će razvijenoj klijentskoj Web aplikaciji omogućiti Bearer token preko Microsoft-a, kako bi razvijena Web aplikacija korišćenjem Bearer token-a mogla da povuče informacije o svim korisnicima određene grupe HTTP upitom na Microsoft Graph API, zatim poslati mail odabranom korisniku.

\subsection{Registrovanje aplikacije na Azure portalu}

Svako ko ima poslovni ili privatni Microsoft profil može da se registruje na Microsoft Azure portal i registruje svoju Web aplikaciju (Slika 1).

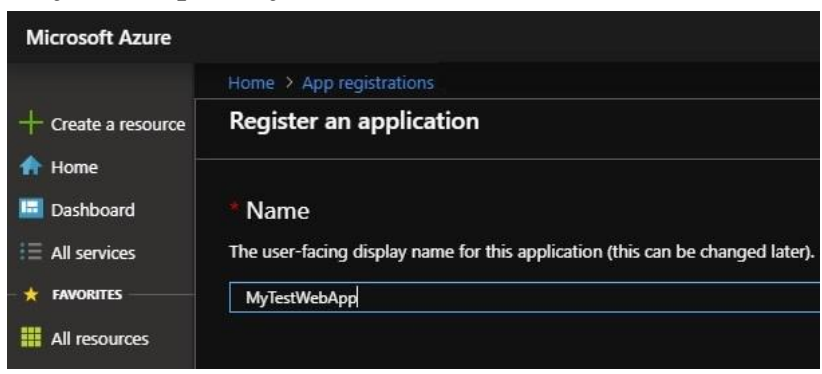

Slika 1. Registrovanje nove aplikacije na Azure

Prilikom registrovanja aplikacije, automatski se dodeljuje identifikator aplikacije od strane Microsoft-a. Za dobavljanje Bearer token-a potrebni su application id koji se automatski generiše, domain (tenant) id (Slika 2) koji se može videti nakon prijave korisnika na Azure portal i Client secret lozinka koju korisnik sam mora da generiše (Slika 3).

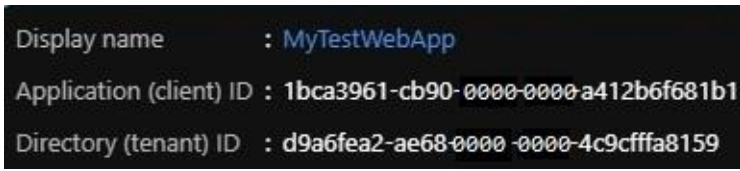

Slika 2. Client ID i Tenant ID

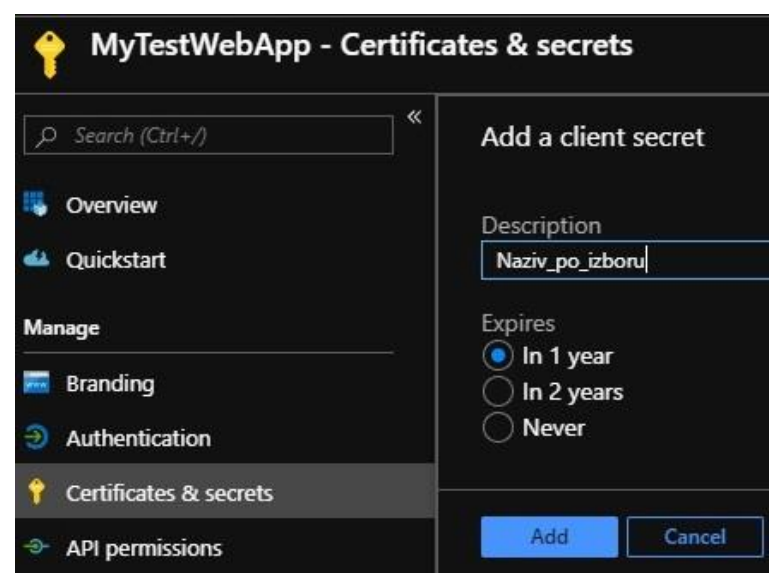

Slika 3. Generisanje Client secret lozinke 
Postoje Microsoft Graph permisije koje omogućavaju korišćenje Microsoft Graph API-a i koje se dele na aplikativne permisije (application permissions) i delegirane (delegated permissions). Aplikativne permisije su za klijente, a delegirane za korisnike. Kako je registrovana aplikacija na Azure portalu klijent, trebaju da joj se dodele aplikativne permisije za čitanje svih korisnika određene grupe (Slika 4) i aplikativne permisije za slanje mail-a umesto bilo kog korisnika (Slika 5).

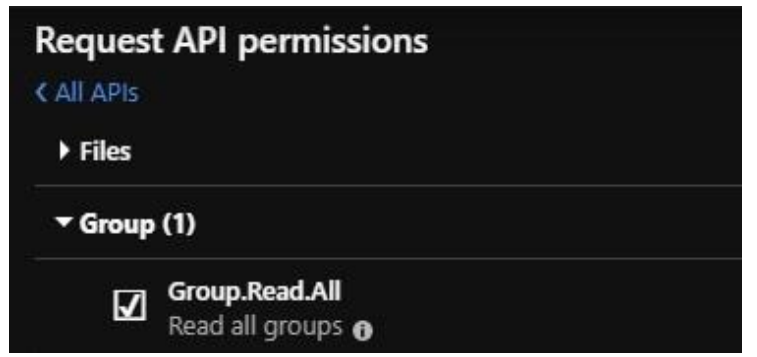

Slika 4. Aplikativne permisije za čitanje podataka grupe

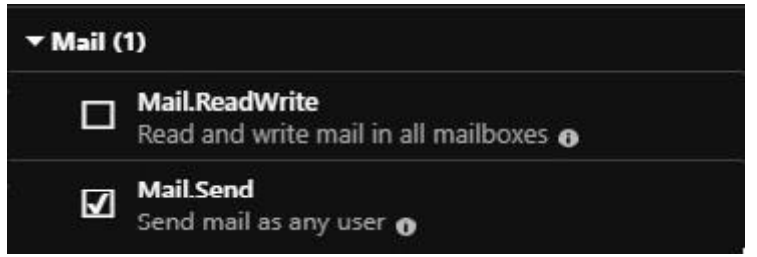

Slika 5. Aplikativne permisije za slanje mail-a kao klijent

Nakon odabira permisija, iste moraju biti odobrene od strane administratora (Slika 6).

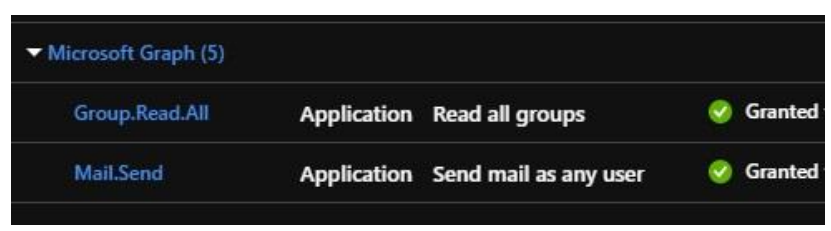

Slika 6. Odobrene aplikativne permisije od strane administratora

\subsection{Dobavljanje Bearer tokena sa Microsoft Exchange servera}

Prilikom pokretanja razvijene Web aplikacije, aplikacija šalje upit na Microsoft preko RESTful servisa putem HTTP POST metode. Za slanje zahteva potrebni su application id od registrovane aplikacije na Azure portalu, Clent secret lozinka i domain id (Slika 7) [7].

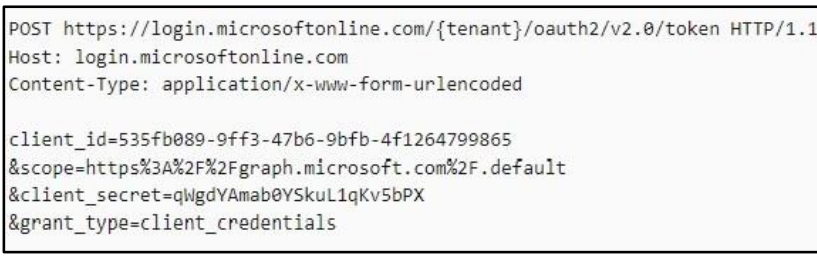

Slika 7. Primer HTTP POST zahteva za dobijanje access Bearer token-a

Aplikacija zatim dobija odgovor o podacima Bearer tokena u JSON formatu gde se može videti koji je tip token-a, kada ističe i sam kriptovan string koji služi za autentifikaciju (Slika 8).

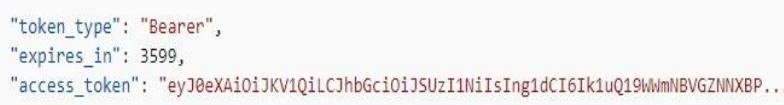

Slika 8. Odgovor na zahtev potraživanja tokena

Token se potražuje ponovo nakon isteka definisanog vremena expires_in u odgovoru.

\subsection{Povlačenje svih korisnika određene grupe pomoću Bearer tokena}

Nakon iniciranja komunikacije od strane razvijene Web aplikacije u cilju definisanja mail-a, automatski se povlače svi korisnici određene grupe iz Active Directory-a tako da se u novootvorenom prozoru klikom na icon „Get Users" može videti lista dobijenih korisnika (Slika 9).

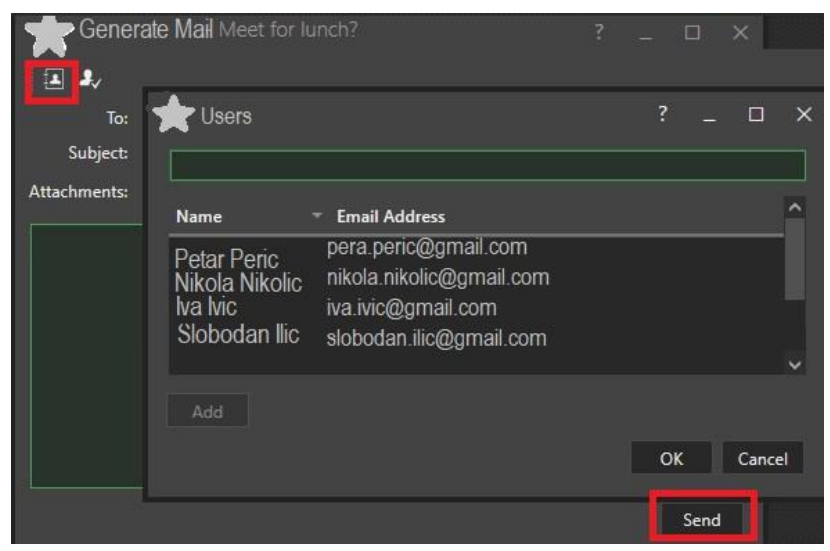

Slika 9. Lista povučenih korisnika iz Active directory-a Prilikom slanja zahteva na Microsoft Graph API u cilju povlačenja svih korisnika određene grupe, kroz Header Authorization zaglavlje se šalje prethodno dobijeni Bearer token, a kroz URL zahteva putem HTTP GET metode šalje se identifikator grupe (Slika 10).

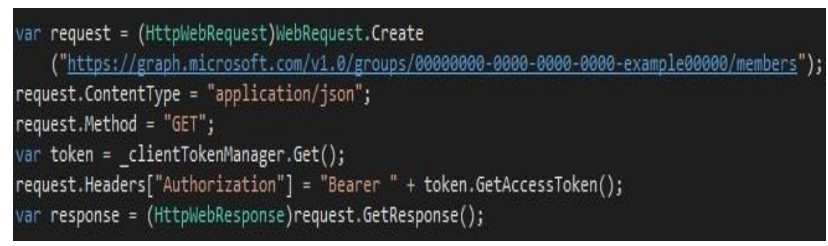

Slika 10. HTTP GET zahtev za dobavljanje korisnika preko Microsoft Graph API-a, sa Bearer autentifikacijom

\subsection{Slanje mail-a odabranim korisnicima pomoću Bearer tokena}

Nakon odgovora od strane Microsoft Graph API-a o potraživanju korisnika određene grupe, krajnji korisnik aplikacije selektuje korisnike kojima želi da pošalje generisani mail. Klikom na dugme „Send“ (Slika 9) inicira se slanje mail-a koje se takođe kao i dobavljanje korisnika vrši preko Microsoft Graph API-a, slanjem HTTP POST zahteva sa definisanim Header Authorization token-om i sadržajem mail-a u body segmentu HTTP POST zahteva. URL HTTP POST zahtev sadrži identifikator korisnika koji šalje mail (Slika 11). 


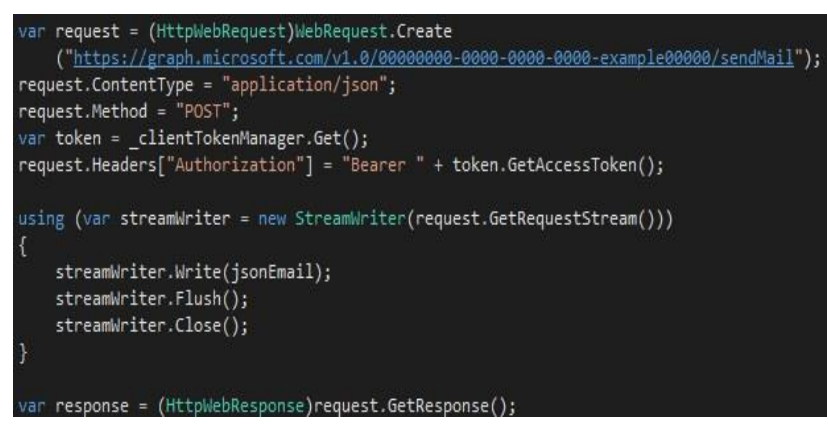

Slika 11. HTTP POST zahtev za slanje mail-a korisnika preko Microsoft Graph API-a, sa Bearer autentifikacijom

\section{ZAKLJUČAK}

U ovom radu opisano je razvijeno rešenje za sigurnu komunikaciju korisnika jednog softvera koji u sklopu svog rešenja ima podršku za slanje mail-a na Microsoft Outlook nalog. Sigurna komunikacija se obavlja uz pomoć Microsoft Bearer token-a koji služi za autentifikovanje klijenta servisu. Za povlačenje tokena sa Microsoft-a, potrebno je poslati HTTP POST zahtev koji sadrži identifikatore koji se dobijaju iz registrovane aplikacije na Azure portalu.

Uslov za povlačenje svih korisnika određene grupe sa Azure Active Directory-a i slanje mail-a u ime željenog korisnika jeste da kompanija svoje podatke o korisnicima (Users) i grupama skladišti na Cloud i da registrovanoj aplikaciji na Azure dodeli aplikativne permisije za čitanje korisnika grupe i slanje mail-a u ime bilo kog korisnika.

Aplikacija koja je realizovana za potrebe povlačenja Bearer token-a i korisnika, kao i za slanje mail-a pisana je u razvojnom okruženju Microsoft Visual Studio 2015.

Predstavljenim rešenjem kompanija oslobađa lokalnu memoriju od čuvanja podataka koje može da skladišti na Cloud i uvodi veću sigurnost podakata kojima ne može svako da pristupi. Pored toga, korisnici mogu centralizovano, samo preko Web aplikacije, da vode svoje aktivnosti.

Kao veći vid sigurnosti razmene podataka na registrovanu aplikaciju se mogu uvesti i sertifikati. Pored čitanja korisnika i slanja mail-a, Microsoft nudi i mnoge druge operacije kao što su čitanje mail-ova korisnika koje mogu biti i po nekom kriterijumu, čitanje osnovnih podataka o kompaniji, praćenje osnovnih događaja kompanije i mnoge druge opcije koje kompanija dozvoli deljenjem podataka na Cloud.

\section{LITERATURA}

[1] Wikipedia, „Microsoft Exchange Server,“, 9 September 2019. Dostupno: https://en.wikipedia.org/wiki/Microsoft_Exchange_Server. [Poslednji pristup Avgust 2019].

[2] Wikipedia, "Wikipedia Outlook", September 2019. Dostupno: https://en.wikipedia.org/wiki/Microsoft_Outlook. [Poslednji pristup Septembar 2019].

[3] Microsoft, „What is Azure Active Directory,“ Microsoft Azure, Dostupno: https://docs.microsoft.com/enus/azure/active-directory/fundamentals/active-directorywhatis. [Poslednji pristup Septembar 2019].

[4] Roy Fielding, Architectural styles and the design of networkbased software architectures. Vol. 7. Doctoral dissertation, California, Irvine: University of California, 2000.

[5] Mumbaikar, Snehal, and Puja Padiya, ,"Web services based on soap and rest principles.", International Journal of Scientific and Research Publications, 2013.

[6] Swagger, „Bearer Authentication,“ 2019. Dostupno: https://swagger.io/docs/specification/authentication/bearerauthentication/. [Poslednji pristup Avgust 2019].

[7] Microsoft, "Get access without a user", Microsoft Graph. Dostupno: https://docs.microsoft.com/en-us/graph/auth-v2service. [Poslednji pristup Septembar 2019].

\section{Kratka biografija}

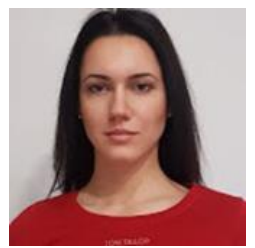

Slađana Dimić rođena je u Novom Sadu 1993. godine. Diplomirala je na Fakultetu tehničkih nauka u Novom Sadu na departmanu za Industrijsko inženjerstvo i menadžment 2016. godine $i$ iste godine upisala master studije na smeru Inženjerstvo informacionih sistema na Fakultetu tehničkih nauka. 